\title{
Subject Index Vol. 54+55, 1990
}

Acetylcholine 368

Adrenal medulla 268,277,312,347

- medullary transplantation 324

- tissue 268 Adriamycin 86 Alcoholism 25

Alzheimer's disease 250, 368, 409 Angiography 471 Apnea 233

Aqua stream and aspirator 457 Arteriovenous malformations 535 Autotransplantation 337

Axoplasmic transport 86

Baclofen 147 Behavior 337 Biopsy 375, 462 Brain atlas 477

- biopsy 409

- edema 364

- grafts 328

- neoplasms 404

- $\quad$ stem injury 233

- transplantation 342

- tumor(s) 193,388,482,501,506,531, 535,541

Carbocyanine 328 Caudate nucleus 277, 324

- _ ultrastructure 297 Cell culture 290

- type 207

Central nervous system 364 Cerebellar afferents 114 Cerebellum 445 
Cerebral blood flow 25

- edema 364

- palsy 130 Cerebrospinal fluid 312

- - catecholamines 306 Cervical injury 233 Chemotherapy 506 Chromaffin cells 250

Chronicpain 80, 168

Computed tomography $25,172,388,404,432,438,493$

- $\quad$ - scan 393

- tomography-guided stereotactic

surgery 453

- - - technique 451 Computer 493

- graphics 482 Craniopharyngioma 525 Creutzfeldt-Jakob disease 409 Cross-

correlation analysis 159 Cryothalamotomy 96

Curie temperature 514 Cyst 525

Dementia 409 Dentatectomy 130 Depth electrodes 60

- recording 193

Digital subtraction angiography 493 Discharge pattern 136 Dopamine 282, 290, 306, 353

- neurons 250 Dorsal root entry zone 80

- - - - surgery 140 Dose distribution 519 Dystonia 159 
Subject Index 
Embryonic tissue transplantation 328 Endoscopic surgery 404 Endoscopy 413 Epilepsy $25,34,56$

- focus 60

- surgery 34 Erythromelagia 96 Essential tremor 125 Evoked potentials 80

Excitotoxicity 21

Fe-Ptalloy 514 Ferromagnetic implant 514 Fetal dopaminergic grafts 290

- tissue 282

Fibrinolysis 445

Functional neurosurgery 125

Gamma unit 535 Ganglionectomy 86 Glioblastoma multiforme 514 Glutamate receptors 21 Glycerol rhizolysis 73 Grafted motoneurons, migration 328 Grafting neurobiology 306 Guided microsurgery 482

Hematoma 445

High-performance liquid chromatography

306 Hippocampal sclerosis 21 Histamine 224 Homovanillic acid 312 Humanbrain 163

- thalamus 186

Hydrocephalus 364

6-Hydroxydopamine 347

Hyperpathia 90

Hypertensive intracerebral hematoma

432,451 Hyperthermia 506 Hypotonia 136

Image processing 477 Imaging 398

Immunocytochemistry 114 Immunological rejection 358 
Implanted electrodes 163 Induction heat ing 514 Intention tremor 172 Interactive device 393 Interstitial hyperthermia 514 Intracavitary irradiation 525 Intracerebral hematoma 427,457

- $\quad$ - evacuation 451

Intracranial electrode 46

Involuntary movements 154

Kufs' disease 409

Laser 398, 501

- hyperthermia 501

- surgery 404 Lexical processing 163 Linear accelerator 519 Lumbar disk surgery 413

Magnetic resonance 51

- $\quad$ - imaging 477,493

- $\quad$ - - scans 393 Malignant tumor 501 Medical Workstation 493 Memory 368

Mental processes 163 Metachromatic leucodystrophy 409 Metastatic brain tumor 514 NMethyl-Z)-aspartate receptors 21 Microcompression 76 Microelectrode(s) 154,168,207

Microneurosurgery 462 Microrecording 168

- rhythmic discharge 186

Microstimulation 168

Microvascular decompression 76

Mixed lymphocyte reaction 358

Models 353

Monkey 337

Mosaic reconstruction 358

Motor control 136

- cortex 34

- nuclei 114 Movement disorders 168 Movement-related cortical potentials 193 MPTP 337 
Subject Index

Nerve growth factor 250

- injury 90

- pathophysiology 90 Neural transplantation 358

- transplants 306 Neuralgia 86

Neuromodifying agents 342 Neuronal activity 163

- circuits 114 Neuropathie pain 90 Neuropil 297 Neurostimulators 56 Nigral afferents

114 Nomenclature 1 Nucleus caudatus 56

- dentatus 56

Operative equipment 457

Pain 73, 86, 90, 96

Pallidotomy 172

Parkinsonism 342

Parkinson's disease 172, 193, 207, 250,

268, 277, 290, 297, 306, 312, 324, 337,

347, 353 Percutaneous procedures 73 Peripheral neurotomies 140 Phrenic pacing 233

Pick's disease 409 Picture-archiving and communication

systems 477 Pontine hemorrhage 453 Positron emission tomography 388 Postoperative dysnomia 46 Postsynaptic involvement 297 Programmable pumps 147 Putamen 277

Quadriplegia 233

Radiation enhancement 531 Radiofrequency thermocoagulation 76 Radiosurgery 375, 519, 535, 541 Radiotherapy 531 Robot 462, 468 Rotationrat 342 
Seizure focus localization 60

Sensorimotor integration 159

Serotonin 364

Single-unit recording 154

Skeletal muscle tone 136

Sonic digitizer 393

Spasticity 80, 140, 147

Speech 34

Speech-dominant temporal lobe 34

Spinal cord 80

- $\quad$ - grafts 328

- $\quad$ - stimulation 224

- infusion 147

Stereo-electroencephalography 51 Stereoencephalotomy 136 Stereoscopic imaging 471

Stereotactic aspiration surgery 432

- coordinates 51

- devices 398

- evacuation 445

- grafting 250

- guidance 51

- neurosurgery 493

- planning 471

- $\quad$ surgery $56,60,96,130,154,172,186$,

193, 207, 250, 268, 277, 282, 375, 388,

$393,404,409,413,427,438,457,462$,

$482,501,506,514,525,531,535,541$

- technique(s) 125,468

Stereotaxis 398

Stereotaxy 268,519

Stroke 159

Subduralgrid 34

SubstanceP 224

Superior cervical ganglion 337 Surgery 80

Surgical computer simulation 488 Synaptology 114

Talairach system 51 Temporal lobe 34,60

- - epilepsy 46

- lobectomy 46 Thalamic hematoma 438

- targets 130

Thalamodentate ablation 130 Thalamotomy 172, 207 
Subject Index 
Thalamus $1,114,168$

- single-unit analysis 159 Therapeutic brain stimulation 56 Three-dimensional imaging 471

- reconstruction 488,541

Tissue transplantation $268,277,282$,

312,324,353,364 Transplantation 268, 347, 368 Trauma 25 Tremor 186,207

Trigeminal neuralgia 73,76 Tyrosine hydroxylase 312,353 
Vasculature 358

Vasoactive intestinal polypeptide 224

Ventral nuclei 1

Ventriculography 51

Ventrolateral thalamotomy 193

VLnucleus 154

Voluntary movement(s) 154,193

Workstation, surgical 393

Xenograft 358 